\title{
EINE UNTERSUCHUNG DER MARKTSITUATION IM BEREICH DER FTI-EVALUIERUNG IN ÖSTERREICH
}

\author{
JÜRGEN STREICHER, WOLFGANG POLT UND MAXIMILIAN UNGER \\ DOI: 10.22163/fteval.2020.472
}

\section{INHALTSVERZEICHNIS}

$\begin{array}{ll}1 & \text { Einleitung } \\ 1.1 & \text { Hintergrund und Zielsetzung } \\ 1.2 & \text { Aufbau und Methodik } \\ 2 & \text { Entwicklung und Status Ouo Der FTI- } \\ & \text { Evaluierung und -Praxis in } \\ 2.1 & \text { Funktion von Evaluierungen im FTI-Bereich } \\ 2.2 & \text { Rechtliche Rahmenbedingungen } \\ 3 & \text { bei Vergabeverfahren } \\ 3.1 & \text { Zentrale Befunde zur Marktsituation im } \\ 3.2 & \text { Bereich FTI-Evaluierung in Österreich } \\ 3.3 & \text { Wettbewerbssituation } \\ 3.4 & \text { Vergabepraxis } \\ 4 & \text { Zusammenfassung und Fazit } \\ 5 & \text { Referenzen } \\ 6 & \text { Abbildungsverzeichnis } \\ 7 & \text { Tabellenverzeichnis }\end{array}$

\section{EINLEITUNG}

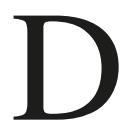

iese Studie untersucht die Marktsituation in Bezug auf Evaluierungen im Bereich Forschungs-, Technologie und Innovationspolitik (im Weiteren. FTI-Evaluierungen) in Österreich. Aufbauend auf einer kurzen Darstellung von Entwicklung und Status quo der FTI-Evaluierungskultur in Österreich beleuchtet sie insbesondere die Entwicklung dieses Evaluierungsmarktes, die Marktteilnehmerlnnen, die Wettbewerbssituation sowie Vergabepraxis. Die Arbeit schließt mit einer Zusammenfassung der wichtigsten Befunde sowie Schlussfolgerungen für die Praxis der FTI-Evaluierung in Österreich.

\subsection{HINTERGRUND UND ZIELSETZUNG}

Evaluierungen bzw. evaluierungs-ähnliche Untersuchungen (z.B. Wirkungsanalysen) im Bereich Forschungs-, Technologie und Innovationspolitik (FTI-Politik) sind heute ein häufiger, z.T. auch gesetzlich vorgeschriebener Bestandteil des politischen Prozesses in Österreich. Die Häufigkeit und Qualität von FTI-Evaluierungen hat in den letzten zwei Jahrzehnten deutlich zugenommen. Teilweise wurde in den letzten Jahren aber auch Kritik an der Verfasstheit des österreichischen Evaluierungsmarkts laut. Dieser sei auf zu wenige Anbieter konzentriert, zu wenig international und methodisch zu wenig innovativ. Mangels systematischer Untersuchungen beruhten solche Einschätzungen jedoch hauptsächlich auf subjektiven Einzelwahrnehmungen.

Die Beschaffenheit des österreichischen Marktes für FTI-Evaluierungen war bislang kaum Gegenstand empirisch fundierter Studien. Zwar gibt es Metaevaluierungen zur österreichischen Evaluierungspraxis. Diese fokussieren jedoch auf Art, Methoden, Qualität und Nutzen der untersuchten Evaluierungen (siehe z.B. Landsteiner 2015, Böttcher et al. 2014, Dinges und Schmidmayer 2010). Sie streifen nur am Rande die Beschaffenheit des österreichischen Evaluierungsmarktes, d.h. die Struktur von Auftraggebern und Auftragnehmern (siehe Reiner und Smoliner 2012).

Ziel der vorliegenden Untersuchung ist es, diese "terra incognita" zumindest teilweise zu erkunden. Im Mittelpunkt stehen Fragen über die Marktgröße bzw. das Marktvolumen, die Marktstruktur sowie der Charakteristika der Marktteilnehmerlnnen. Darüber hinaus wurden auch die Einschätzungen der Marktteilnehmerlnnen hinsichtlich der Markt- bzw. Wettbewerbssituation sowie relevanter Entwicklungen erhoben. 
Die vorliegende Untersuchung soll eine empirische Grundlage für weiterführende Diskussionen schaffen. Naturgemäß konnte sie aufgrund von Zeit- und Ressourcenbeschränkungen nicht alle Aspekte des FTI-Evaluierungsmarktes ausleuchten. So ist etwa der Innovationsgehalt der in Österreich verwendeten Evaluierungsmethoden nicht Gegenstand der vorliegenden Untersuchung gewesen. Ob Marktdynamiken in Österreich anders als in vergleichbaren Ländern verlaufen, konnte mangels Vergleichsdaten ebenfalls nicht festgestellt werden. Es konnten auch nicht alle Aspekte der Wettbewerbsintensität ausgeleuchtet werden. Deshalb wären etwa evaluierungs-ähnliche Untersuchungen, die Erfassung von MarktteilnehmerInnen außerhalb der Plattform fteval oder Vergleiche auf internationaler und regionaler Ebene weitergehende Analysen wert.

\subsection{AUFBAU UND METHODIK}

Die vorliegende Arbeit untersucht die Marktsituation im Bereich der FTI-Evaluierung in Österreich. Der Fokus liegt also auf dem Bereich Forschung, Technologie und Innovationspolitik (FTI-Politik). Aufgrund der manchmal großen inhaltlichen Nähe zur Hochschulpolitik und zu den damit verbundenen Maßnahmen wurden auch relevante Evaluierungen aus diesem Bereich in die Betrachtungen miteinbezogen.

Bei der Definition des Begriffs „Evaluierung" wurde auf die Standards der Deutschen Gesellschaft für Evaluation e.V. zurückgegriffen. Unter einer Evaluierung bzw. Evaluation ${ }^{1}$ wird demnach die „systematische Untersuchung von Nutzen und/oder Güte eines Gegenstands (Evaluationsgegenstand) auf Basis von empirisch gewonnenen Daten ... Iverstanden. Sie impliziert] ... eine Bewertung anhand offengelegter Kriterien für einen bestimmten Zweck." (DeGEval Standards 2016, S. 33). Diese Definition wird auch in den Evaluierungsstandards der Plattform fteval verwendet (fteval Standards 2019, S. 5-6).

Der Untersuchungszeitraum umfasst die zehn Jahre von 2009 bis 2018. Um in die Untersuchung miteinbezogen zu werden, muss bzw. musste der Evaluierungsgegenstand (z.B. ein Programm) einen möglichst klaren Bezug zur FTI-Politik aufweisen - insbesondere im Titel der Untersuchung - sowie auf Bundesebene implementiert sein. Zwar ist es auch möglich, eine Vielzahl von anderen Materien zu evaluieren (Gesetze, Strategien, Richtlinien, Prozesse, Technologien, Dienstleistungen, Veranstaltungen, Schulungen, Materialien, Berichte u.a., vgl. fteval Standards 2019, S. 12) - die entsprechende Berücksichtigung dieser Gegenstände hätte jedoch den Rahmen der gegenständlichen Arbeit gesprengt.

Kapitel 2 bietet einen kurzen Überblick über die Entwicklungen und den Status quo der FTI-Evaluierungskultur und -praxis in Österreich. Dabei werden Funktionen sowie die rechtlichen Rahmenbedingungen von Evaluierungen im FTI-Bereich sowie die rechtlichen Rahmenbedingungen beschrieben. Kernstück der Studie bilden die empirischen Befunde, welche auf Basis von Sekundärdaten (Überblick vgl. Tab. 1) sowie einer Befragung von Marktteilnehmerlnnen gewonnen wurden (siehe Kapitel 3).

\begin{tabular}{|l|l|}
\hline 2009-2018 & Repository der Plattform für Forschungs- und \\
& Technologiepolitikevaluierung (fteval) \\
& Veröffentlichungen der \\
& Bundesforschungsdatenbank (bfdat) \\
& Internetauftritte der relevanten Ministerien und \\
& Förderagenturen sowie öffentliche Mittler (auftrag. \\
& at, Bundesbeschaffung GmbH) und Evaluierungs- \\
& Anbieter in Österreich (fteval Mitglieder) \\
\hline 2014-2018 & $\begin{array}{l}\text { Beantwortung parlamentarischer Anfragen } \\
\text { betreffend die Erbringung von Dienstleistungen } \\
\text { für das BMBWF, BMVIT und BMDW bzw. } \\
\text { relevante Vorgängereinrichtungen } \\
\text { Vergabebekanntmachungen im „Supplement } \\
\text { zum Amtsblatt der Europäischen Union } \\
\text { (Tenders Electronic Daily, TED) }\end{array}$ \\
\hline
\end{tabular}

Tabelle 1: Verwendete Quellen von Sekundärdaten

Auf Basis der Sekundärdaten wurde die Anzahl von Evaluierungen im FTI-Bereich von 2009 bis 2018 recherchiert. Evaluierungen, die in mehreren Schritten durchgeführt wurden bzw. mehrere zusammenhängende Berichte umfassten, wurden dabei als eine Untersuchung gewertet. Die verschiedenen Informationen zu den einzelnen Evaluierungen, z.B. Titel, Abschlussjahr, Auftragnehmerlnnen etc. wurden in einer Liste zusammengeführt und den identifizierten Auftraggeberlnnen mit der Bitte um Vervollständigung zur Verfügung gestellt.

Im Zeitraum Mai bis Juni 2019 wurden die Mitgliedseinrichtungen der Plattform fteval gebeten, an einer Online-Befragung, die sich jeweils an AnbieterInnen bzw. Auftraggeberlnnen von Evaluierungen richtete, teilzunehmen. Bei Anbietern lag der Schwerpunkt dieser Erhebung auf dem Umfang ihrer Tätigkeiten im Evaluierungsbereich sowie auf dem Stellenwert dieses Bereichs in ihrem Leistungsportfolio. Bei Nachfragern lag der Schwerpunkt auf der Anzahl eingelangter Bewerbungen und vergebener Aufträge sowie auf den vergebenen Volumina. Die Fragen bezogen sich überwiegend auf die letzten drei Jahre (2016 bis 2018). Die erhobenen Inhalte wurden mit den Ergebnissen der Sekundärdatenanalyse abgeglichen sowie mit den Einschätzungen von Mitgliedern der Plattform fteval im Rahmen einer Diskussionsveranstaltung ${ }^{2}$ kontrastiert.

Als für die Befragung relevante Marktteilnehmerlnnen wurden 13 potentielle Auftragnehmer und zehn potentielle Auftraggeberlnnen identifiziert. Diesen wurden Links zur Online-Befragung zugesandt. Die Versendung erfolgte nicht durch die JOANNEUM RESEARCH sondern durch eine Rechtsanwaltskanzlei. Dies sicherte die Anonymität der Angaben und gewährleistete, dass die Studienautoren keinen Einblick in die Situation einzelner MitbewerberInnen erhielten. Von den so kontaktierten Markteilnehmern übermittelten 12 Auftragnehmer und acht AuftraggeberInnen einen auswertbaren Fragebogen. Der Rücklauf lag somit bei rund $90 \%$ bzw. $80 \%{ }^{3}$ Die Studienautoren möchten an dieser Stelle den Mitgliedern der Plattform fteval nochmals herzlich für die Teilnahme an der Befragung danken.

$1 \quad$ Die Begriffe Evaluierung und Evaluation wurden im Rahmen der Sekundärdatenanalyse und Primärerhebung synonym verwendet. Die vorliegende Arbeit wählt Evaluierung als primäre Bezeichnung.

2 „Der Evaluierungsmarkt im FTI-Bereich in Österreich. Ergebnisse der Marktstudie im Auftrag der Österreichischen Plattform für Forschungs- und Technologiepolitikevaluierung". Veranstaltung der Plattform fteval, 5. Juli 2019, Wien.

3 Rücklauf Auftraggeberlnnen: Neun, auswertbar waren acht: Aufgrund der geringen Überlappung von durchführenden bzw. beauftragenden Einrichtungen lässt sich abschließend nicht klären, ob nicht doch eine Vollerhebung stattgefunden hat. 
Bezüglich der Analyse der Befragungsergebnisse ist zu beachten, dass aufgrund der Zusammensetzung der Mitgliedseinrichtungen der Plattform fteval zwar eine repräsentative, aber kleine Samplegröße ${ }^{4}$ zur Verfügung stand, sodass deskriptive Auswertungen gewissen Verzerrungen durch Ausreißer unterliegen können.

In einem abschließenden Arbeitsschritt wurden die Ergebnisse zusammengeführt und einer analytischen Betrachtung unterzogen (siehe Kapitel 4).

\section{ENTWICKLUNG UND STATUS QUO DER FTI- EVALUIERUNGSKULTUR UND -PRAXIS IN ÖSTERREICH}

Evaluierungen haben sich als Instrument im Prozess der FTI-Politik international etabliert. Sie ermöglichen strukturiertes Feedback und die Bereitstellung politikrelevanten Wissens, nicht nur für die Planung, Abwicklung und Bewertung bestehender Maßnahmen. Sie schaffen auch verbesserte Grundlagen für die Entwicklung von Ideen und die Erarbeitung neuer Initiativen. Nicht zuletzt im Kontext der europäischen FTIPolitik steigt der Informations- und Lernbedarf über die Funktionsweise des österreichischen Forschungs- und Innovationssystems, die Qualität der österreichischen Forschung und Entwicklung und die Wirksamkeit von FTI-Förderung im internationalen Vergleich.

\subsection{FUNKTION VON EVALUIERUNGEN IM FTI-BEREICH}

Vor diesem Hintergrund sind in den letzten zwei Jahrzehnten die FTI-Evaluierungstätigkeiten in Österreich stark angestiegen. Evaluierungskompetenzen und -kapazitäten im öffentlichen Bereich wurden ausgebaut. Entsprechende Zuständigkeiten in Ressorts und Agenturen wurden geschaffen. Österreich liegt heute hinsichtlich der Anzahl von Evaluierungen im FTI-Bereich im europäischen Spitzenfeld. Studien, die sich dem Umgang mit Evaluierungen widmen, unterstreichen die im Allgemeinen hohe Professionalität und Qualität österreichischer Evaluierungen (vgl. Tsipouri und Sidiropolous 2014, Dinges und Schmidmayer 2010; siehe auch Reiner und Smoliner 2012, BMWFW und BMVIT 2017). Andererseits ist eine zunehmende Institutionalisierung und Routinisierung zu beobachten, mit Auswirkungen auf das Nutzen von und das Lernen aus Evaluierungen (vgl. Streicher 2017, Landsteiner 2015, Biegelbauer 2013).

Die österreichische Evaluierungspraxis im FTI-Bereich spiegelt die Struktur des Forschungsförderungssystems wider. Diese Evaluierungs- praxis ist von Evaluierungen zahlreicher, relativ kleiner einzelner Programme und Maßnahmen geprägt. Institutionen, Organisationen oder gesetzliche Regelungen werden dagegen selten evaluiert. Evaluierungen nehmen überwiegend eine formative Perspektive ein und finden in bestimmten, oft gesetzlich vorgeschriebenen, Intervallen während der Laufzeit einer Intervention statt (sogenannte Interims- oder Zwischenevaluierungen). Ex-ante Evaluierungen, begleitende Evaluierungen bei erstmaliger Umsetzung und summative Evaluierungen sind weniger häufig anzutreffen.

Evaluierungen fungieren als wichtiges Instrument der FTI-Politik und Verwaltungsführung, das dazu beiträgt, Transparenz, Rechenschaftslegung und eine evidenzbasierte Entscheidungsfindung zu unterstützen. Die Durchführung erfolgt in Österreich auf der Basis allgemeiner gesetzlicher Erfordernisse, spezifischer Anforderungen im Kontext von Richtlinien und Fördertätigkeiten sowie haushaltrechtlicher Maßgaben. Die Rechtsgrundlagen wurden in den letzten Jahren laufend aktualisiert, Begrifflichkeiten angepasst sowie Regelungen überarbeitet. Zu den maßgeblichen Gesetzen und Vorschriften zählen das Forschungs- und Technologieförderungsgesetz (FTF-G), die Allgemeinen Rahmenrichtlinien für die Gewährung von Förderungen aus Bundesmitteln (ARR 2014), das Forschungsorganisationsgesetz (FOG; Berichtswesen: §§ 6-9) sowie die auf diesen Gesetzen basierenden Richtlinien zur Forschungsförderung und zur Förderung ${ }^{5}$ der wirtschaftlich-technischen Forschung, Technologieentwicklung und Innovation (die sogenannten FTI-Richtlinien). ${ }^{6}$ Im Einklang mit der Implementierung der wirkungsorientierten Haushalts- und Verwaltungsführung wurden 2015 die Richtlinien überarbeitet. Dabei wurde eine Fokussierung auf inhaltliche Ziele und Indikatoren vorgenommen (vgl. BMBWF, BMVIT und BMDW 2018).

Zudem entwickelte sich in den letzten Jahren ein reger Diskurs über Möglichkeiten, Funktion und Nutzen von Evaluierungen, Ansprüche an und den Umgang mit Evaluierungen (vgl. BMWFW und BMVIT 2017, siehe auch: Warta und Philipp 2016). Es hat sich rund um die Evaluierungspraxis eine Community mit einer "gemeinsamen Sprache" entwickelt. Dort wird über Themen wie Wirkungsorientierung und Folgenabschätzung debattiert. Die Plattform fteval stellt dabei seit Jahren ein wichtiges Forum dar. Sie formuliert und publiziert Standards der Evaluierung in der Forschungs- und Technologiepolitik (vgl. fteval Standards 2019).

\subsection{RECHTLICHE RAHMENBEDINGUNGEN BEI VERGABEVERFAHREN}

Evaluierungen stellen aus der Sicht der öffentlichen Hand einen Beschaffungsfall dar. Sie werden als Bestellungen der öffentlichen Hand im Rahmen der öffentlichen Auftragsvergabe realisiert. In Österreich können öffentliche Aufträge durch Bedarfsbündelung der öffentlichen unterschiedlichem Nachfrageverhalten nach Evaluierungen zusammensetzen.

5 Vgl. Richtlinien der Bundesregierung über die Gewährung und Durchführung von Förderungen gemäß §§ 10-12 F0G, BGBI. Nr. 341/1981

$6 \quad$ Vgl. Richtlinien zur Förderung der wirtschaftlich-technischen Forschung, Technologieentwicklung und Innovation (FTI-Richtlinie 2015); das sind: Themen-FTIRichtlinie, Struktur-FTI-Richtlinie, Humanressourcen-FTI-Richtlinie gemäß Forschungs- und Technologieförderungsgesetz (FTF-G) des Bundesministers für Verkehr, Innovation und Technologie (GZ BMVIT-609.986/0011-III/I2/2014) und des Bundesministers für Wirtschaft und Arbeit (GZ BMWFW-97.005/0003C1/9/2014). 
Einrichtungen zentral oder durch jeden Bedarfsträger selbst, also dezentral, im Rahmen der Gesetze, vergeben werden. ${ }^{7}$ Die zentrale Beschaffung für Bundesdienststellen und andere öffentliche AuftraggeberInnen übernimmt in Österreich seit 2001 die Bundesbeschaffung GmbH (BBG) als Tochter des Bundesministeriums für Finanzen (BMF).

Seit dem Beitritt zur Europäischen Union (EU) 1995 existiert in Österreich für die öffentliche Auftragsvergabe ein gesetzliches Regulativ. Seit 2002 gilt ein einheitliches Bundesvergabegesetz (BvergG). Zuletzt wurde dieses Gesetz 2018 novelliert. Zu den zentralen Grundsätzen des Vergaberechts (§ 20 BVergG 2018) zählen u.a. ein freier und lauterer Wettbewerb, Gleichbehandlung aller Bewerber sowie allgemein die Gewährleistung von Transparenz in Vergabeverfahren.

Das Bundesvergabegesetz sieht in seiner aktuellen Fassung eine Reihe von Vergabeverfahren vor ( $\$ 31$ BVergG 2018). Diese sind: offenes Verfahren, nicht offenes Verfahren mit/ohne vorherige(r) Bekanntmachung, Verhandlungsverfahren mit/ohne vorherige(r) Bekanntmachung, Direktvergabe (auch: mit vorheriger Bekanntmachung), Rahmenvereinbarung, dynamisches Beschaffungssystem, wettbewerblicher Dialog, elektronische Auktion (kein eigenes Vergabeverfahren) und die Innovationspartnerschaft. Weiters lassen sich die Verfahrensarten nach den durchzuführenden Schritten (ein- bis dreistufiges Vergabeverfahren) unterteilen.

Wesentliches Kriterium für die Art der Vergabe ist der geschätzte Auftragswert der zu vergebenden Leistung. Im Vergabeverfahren wird zwischen dem Oberschwellenbereich und dem Unterschwellenbereich unterschieden. Im Oberschwellenbereich kommen nicht sämtliche Verfahrensarten zur Anwendung, und es bestehen auch höhere Anforderungen an die Publizität des Vergabeverfahrens. Ob ein Vergabeverfahren im Oberschwellenbereich oder Unterschwellenbereich vorliegt, orientiert sich am (geschätzten) Auftragswert. Der Grenzwert zum Oberschwellenbereich für öffentliche Liefer- und Dienstleistungsaufträge einer zentralen Beschaffungsstelle sowie vergleichbarer Bundeseinrichtungen beträgt $144.000 €(\S 12$ BVergG 2018). Für alle übrigen Liefer- und Dienstleistungsaufträge beläuft sich der Schwellenwert auf $221.000 €$ (Bauaufträge: 5.548 .000 €). Ab dem Erreichen dieser Grenzwerte muss zusätzlich zur Bekanntmachung im regionalen Amtsblatt eine Meldung an das Amtsblatt der europäischen Union (Tenders Electronic Daily, TED) ergehen.

Im Unterschwellenbereich wurde die Schwellenwerteverordnung von 2009 (VO BGBI. II Nr. 125/2009) im Jahr 2018 bis zum 31. Dezember 2020 neuerlich verlängert. Aufträge im Bau-, Liefer- und Dienstleistungsbereich können weiterhin bis zu einem geschätzten Auftragswert von $100.000 €$ (ohne Ust.) direkt, ggf. nach Einholung von Angeboten oder unverbindlichen Preisauskünften, von einem oder mehreren Interessenten an geeignete leistungsfähige und zuverlässige Auftraggeberlnnen vergeben werden. Anzumerken ist, dass in den relevanten Ressorts verschiedene, mehr oder weniger formalisierte, Verfahren und Vorgangsweisen etabliert wurden, welche die Direktvergabe von geistigen Dienstleistungen unter Berücksichtigung der Grundsätze des Vergaberechts regeln. Dazu gehören u.a. Bestimmungen bezüglich der Einholung und Bewertung von Angeboten.

\section{ZENTRALE BEFUNDE ZUR MARKTSITUATION IM BEREICH FTI-EVALUIERUNG IN ÖSTERREICH}

Im Folgenden werden die wichtigsten Befunde aus der Analyse der Sekundärdaten und der Online-Befragung präsentiert.

\subsection{ENTWICKLUNG UND NACHFRAGE VON EVALUIERUNGEN}

Im Zuge der ersten Recherchephase konnten für den Zeitraum 2009 bis 2018 über 500 Studien, Analysen und Expertisen mit mehr oder weniger starkem Bezug zum Themenfeld FTI identifiziert werden. Unter Berücksichtigung der eingangs beschriebenen Definitionen konnte die Anzahl auf 107 einzelne Evaluierungen eingegrenzt werden. Abb. 1 gibt die Entwicklung der Zahl dieser Evaluierungen im Zeitverlauf wider. Diese zeigt starke Schwankungen bis 2014, was zum einen auf die umfassende Systemevaluierung zu Beginn des Betrachtungszeitraums (Aiginger et al. 2009) und die im Vergleich zu den Folgejahren schwächere Datenbasis zurückgeführt werden kann. Andererseits ist zu vermuten, dass die Sekundärdatenbasis für frühere Jahre schwächer ist, da ein Trend zur vermehrten Darstellung von Referenzprojekten auf den Websites der Anbieter feststellbar ist.

Über den gesamten Zeitraum betrachtet lag die Leitung der Evaluierungen in ca. neun von zehn Fällen bei einer österreichischen Einrichtung. Der Rest verteilt sich auf Einrichtungen aus anderen europäischen Ländern, insbesondere auf solche aus Deutschland. Knapp drei Viertel aller Evaluierungen wurden von Einrichtungen durchgeführt, die mit Stand Juni 2019 auch Mitglied bei der Plattform fteval waren. In einem Drittel der übrigen Fälle waren diese, soweit dies nachvollziehbar war, immerhin als Partner dabei. Der Anteil an fteval-Mitgliedern an den durchgeführten Evaluierungen ist in den letzten Jahren des Beobachtungszeitraums jedoch zurückgegangen. 


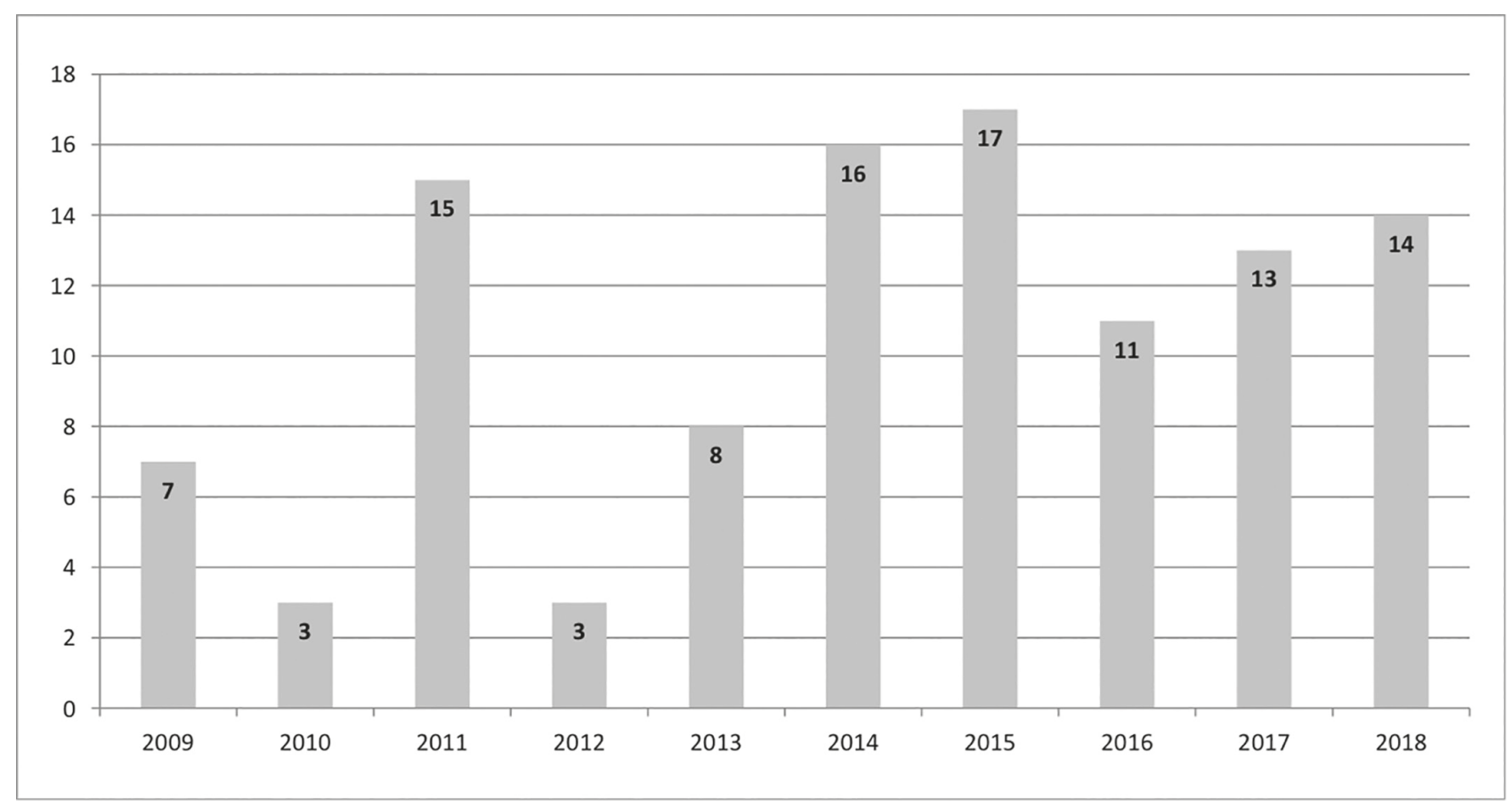

Abbildung 1: Anzahl an identifizierten Evaluierungen im Zeitraum 2009-2018 Quelle: Sekundärdatenanalyse (vgl. Tab. 1).

Ein Vergleich mit einer der frühesten Erhebungen (Dinges und Schmidmayer 2010) zeigt, dass sich die Evaluierungsaktivitäten seit Mitte der 2000er Jahre ca. verdoppelt haben: Wurden zwischen 2002 und 2007 im Schnitt sechs Evaluierungen pro Jahr durchgeführt, so waren es laut Sekundärdatenanalyse im Zeitraum 2016 bis 2018 bereits 13 (vgl. Tab 2).

Laut den AuftraggeberInnen wurden im Untersuchungszeitraum im Schnitt rund 17 Evaluierungen pro Jahr beauftragt, was insgesamt als Untergrenze angenommen werden kann. Die Differenz zum Ergebnis der Sekundärdatenanalyse ist zum Teil auf nicht publizierte Evaluierungen zurückzuführen. Die höchste Anzahl an durchgeführten Evaluierungen (25) im Zeitraum 2016 bis 2018 wird in der Befragung der Auftragnehmerlnnen genannt. Es ist zu vermuten, dass hier auch Arbeiten berücksichtigt wurden, die nicht nur Maßnahmen auf Bundesebene adressieren oder eher dem Spektrum evaluierungs-ähnlicher Untersuchungen zuzuordnen sind - aber durch die Auftragnehmerlnnen als Evaluierungen im Sinne dieser Untersuchung eingestuft wurden.

Mit Blick auf die unterschiedlichen Arten von Evaluierungen (siehe Tab. 2) fällt erwartungsgemäß die Dominanz von Interims- bzw. Zwischenevaluierungen auf. Überraschend ist die von den Auftragnehmerlnnen genannte hohe Anzahl an Ex-ante Evaluierungen. Dieser Befund steht im Kontrast zu bisherigen Beobachtungen. Abseits der oben angeführten Vermutung bezüglich des Umfangs der berücksichtigten Arbeiten dürften verwandte und dennoch unterschiedliche Konzepte (z.B. Roadmaps, Entwicklungsszenarien, Foresight und Technikfolgen-Abschätzungen), die zuletzt verstärkt Einsatz im FTI-Bereich fanden (vgl. BMWFW und BMVIT 2017) - in der Befragung als Ex-ante Evaluierungen eingeordnet worden sein.

\begin{tabular}{|c|c|c|c|c|}
\hline \multirow{2}{*}{ Evaluierungsart ${ }^{1}$} & \multicolumn{4}{|c|}{ Durchschnitt pro Jahr } \\
\hline & $2002-2007^{\mathrm{a}}$ & $2016-2018^{b}$ & $2016-2018^{c}$ & $2016-2018^{d}$ \\
\hline Ex-ante Evaluierungen ${ }^{2}$ & 0,8 & 0,3 & 0,7 & 4,3 \\
\hline Interims-/Zwischenevaluierungen ${ }^{3}$ & 0,7 & 10,7 & 7,3 & 12,0 \\
\hline Begleitende Evaluierungen ${ }^{4}$ & 3,0 & 1,0 & 3,3 & 4,3 \\
\hline Ex-post Evaluierungen (inkl. andere) ${ }^{5}$ & 1,2 & 0,7 & 5,3 & 4,3 \\
\hline Summe & 5,7 & 12,7 & 16,7 & 25,0 \\
\hline
\end{tabular}

Tabelle 2: Evaluierungen auf nationaler Ebene, 2002-2007 sowie 2016-2018

Anmerkungen: a Dinges und Schmidmayer 2010, b Sekundärdatenanalyse, c Befragung Auftraggeberlnnen, d Befragung Auftragnehmerlnnen; 1 Definitionen laut fteval Standards 2019, S. 12; vergleichbar mit den Definitionen in Dinges und Schmidmayer 2010, S. 253. ${ }^{2}$ setzen vor Beginn einer Intervention an und haben einen zukunftsgerichteten Charakter; ${ }^{3}$ durchgeführt während der Laufzeit einer Intervention, unterschiedliche Foki; ${ }^{4}$ setzen zu Beginn einer Intervention an und erfolgen parallel zu Ihrer Umsetzung, unterschiedliche Foki; ${ }^{5}$ direkt und/oder lange nach Abschluss einer Intervention, inkl. „terminale“ Evaluierungen zum Maßnahmenende bzw. „other” laut Dinges und Schmidmayer 2010. 
Summiert über alle befragten Auftragnehmerlnnen, geben diese an, zwischen 2016 und 2018 im Schnitt pro Jahr ca. $750.000 €$ durch FTIEvaluierungen eingeworben zu haben. Auftraggeberlnnen schätzen das Volumen an extern vergebene Evaluierungen für denselben Zeitraum im Jahresdurchschnitt auf ca. $760.000 €$. Die Einschätzungen von Auftraggeberlnnen und Auftragnehmerlnnen zur Marktreife decken sich also in hohem Maße. Die Ergebnisse der Sekundärdatenanalyse sind diesbezüglich unvollständig, weisen aber tendenziell in dieselbe Richtung.

Im Einklang mit den Befunden der Sekundärdatenanalyse geben $58 \%$ (sieben von zwölf) der befragten Auftragnehmerlnnen an, dass die Nachfrage gemessen am Gesamtvolumen der ausgeschriebenen Evaluierungsaufträge im FTI- Bereich im Inland zwischen 2016 und 2018 unverändert geblieben ist (für $25 \%$ zugenommen, für $17 \%$ abgenommen). Laut $63 \%$ (fünf von acht) der befragten AuftraggeberInnen war im selben Zeitraum in ihren Einrichtungen die Nachfrage nach Evaluierungen unverändert (für 37 \% zugenommen). Zuletzt entwickelte sich die Nachfrage nach Evaluierungen eher gleichmäßig, was sich mit jüngsten Beobachtungen über die Regelmäßigkeit und Routine des Evaluierungsaufkommens im FTI-Bereich in Österreich deckt (Streicher 2017, Landsteiner 2015).

\subsection{MARKTTEILNEHMER UND MARKTTEILNEHMERINNEN}

Institutionell wird das Politikfeld FTI vor allem durch drei Ministerien bestimmt: (1) Bundesministerium für Bildung, Wissenschaft und Forschung (BMBWF), (2) Bundesministerium für Digitalisierung und Wirtschaftsstandort (BMDW) und (3) Bundesministerium für Klimaschutz, Umwelt, Energie, Mobilität, Innovation und Technologie (BMK). Diese bzw. relevante Vorgängereinrichtungen sind, oft gemeinsam auftretend, aktuell die wesentlichen AuftraggeberInnen für Evaluierungen auf Bundesebene. Die Sekundärdatenanalyse bestätigt dies. Weitere relevante Ressorts für die Vergabe von FTI-Evaluierungen, die jedoch nicht befragt wurden, können das Bundesministerium für Finanzen (BMF) und das Bundesministerium für Soziales, Gesundheit, Pflege und Konsumentenschutz (BMSGPK) sein. Auch das Bundesministerium für Landwirtschaft, Regionen und Tourismus (BMLRT) und das Bundesministerium für Landesverteidigung (BMLV) beauftragen gelegentlich Evaluierungen bzw. evaluierungs-ähnliche Untersuchungen mit Forschungs- und/oder Technologieinhalten.

Die Gegenstände von Evaluierungen - oft FTI-Programme - werden zumeist von Agenturen im Auftrag eines Trägers/Ministeriums umgesetzt. Diese Agenturen sind im Bereich der angewandten Forschung die Forschungsförderungsgesellschaft (FFG) sowie das Austrian Wirtschaftsservice (aws). Im Bereich der Grundlagenforschung ist es der Fonds zur Förderung der wissenschaftlichen Forschung (FWF). Laut Sekundärdatenanalyse tritt der FWF für Evaluierungen, die seine Programme und Maßnahmen betreffen, regelmäßig auch als Auftraggeber auf. Im Gegensatz dazu ließen sich nur wenige Evaluierungen in den Sekundärdatenquellen finden, die von FFG oder aws beauftragt wurden.

Die befragten AnbieterInnen von Evaluierungen unter den ftevalMitgliedsorganisationen, welche auch am Großteil der Evaluierungen der letzten Jahre beteiligt waren, setzen sich aus unterschiedlichen Forschungsorganisationen und Beratungsunternehmen zusammen (vgl. Warta und Philipp 2016). Laut Befragung stuft sich die Hälfte (sechs von zwölf) als private außeruniversitäre Forschungseinrichtung ein, der Rest ordnet sich öffentlichen außeruniversitären Forschungseinrichtungen, privatwirtschaftlichen Unternehmen sowie sonstigen Einrichtungen zu. Gemessen am Gesamtumsatz zeigt sich eine relativ gleichmäßige Verteilung der Einrichtungen über ein Spektrum von „kleinen“ mit weniger als $500.000 €$ bis hin zu "großen“ Einrichtungen mit mehr als 5 Mio. $€$ Gesamtumsatz pro Jahr, es sind also Einrichtungen unterschiedlichster Größe auf dem österreichischen Markt vertreten.

Die Auftragnehmerlnnen sind vielfach international aktiv: Zwei Drittel (acht von zwölf) geben an, in ausgewählten europäischen Ländern tätig zu sein. Für mehr als die Hälfte stellt die gesamte EU einen Markt dar. Auch die Themenbereiche, in welchen zwischen 2016 und 2018 Evaluierungen angeboten bzw. durchgeführt wurden, sind breit gestreut. Kaum abgedeckt werden durch die hier erfassten Institutionen aber Themen der internationalen Entwicklung oder der Kultur und Kulturpolitik.

Zu den am häufigsten genannten evaluativen Tätigkeitsschwerpunkten der befragten Einrichtungen zählen die Evaluierung von Förder- und Forschungsprogrammen sowie die Erstellung von Studien, die in Teilen einen Evaluierungscharakter aufweisen (jeweils 80 \%, zehn von zwölf). Bei der Evaluierung von Einrichtungen bzw. Institutionen beträgt der Anteil $75 \%$ (neun von zwölf), bei der Durchführung von Wirkungsanalysen $67 \%$ (neun von zwölf). Reviews und Gutachten werden relativ selten als Tätigkeitsschwerpunkt genannt.

Nur wenige Einrichtungen bilden das gesamte Spektrum an evaluativen Tätigkeiten ab. Viele haben ein über die Jahre entwickeltes Set an Tätigkeitsschwerpunkten und können auf Erfahrung in mehreren Themenbereichen aufbauen. Nahezu alle befragten Auftragnehmerlnnen sind zudem in den letzten Jahren im Zuge von FTI- Evaluierungen eine Kooperation mit anderen Partnern eingegangen.

Für die befragten Auftragnehmer spielen FTI-Evaluierungen im Rahmen ihrer Geschäftstätigkeit eine wichtige, jedoch nicht die Hauptrolle. 2018 lag der Anteil an entsprechenden Evaluierungen am Gesamtbudget bzw. -umsatz bei $27 \%$ (im Inland vergeben) bzw. $15 \%$ (im Ausland vergeben). Für die nächsten Jahre (bis 2023) wird eine leichte Verschiebung der Anteile zugunsten von Evaluierungen, die im Ausland vergeben werden, erwartet. Die erwirtschafteten Erträge aus Evaluierungsaufträgen werden als überwiegend kostendeckend eingeschätzt $(75 \%$, neun von zwölf). Ein Viertel gab jedoch auch an, dass Evaluierungen, die auf nationaler Ebene vergeben wurden, in Summe nicht kostendeckend durchgeführt werden konnten.

\subsection{WETTBEWERBSSITUATION}

Die Ausschreibung und Vergabe von Aufträgen im Bereich der FTIEvaluierungen in Österreich sehen 67 \% (acht von zwölf) der befragten Auftragnehmerlnnen als stark auf wenige AuftraggeberInnen konzentriert. Dabei verfügen lediglich $25 \%$ (drei von zwölf) über Schlüsselauftraggeber bzw. Stammkunden. Bei 42 \% (fünf von zwölf) wechseln die AuftraggeberInnen häufig, bei 33 \% (vier von zwölf) zumindest gelegentlich. Etwas anders stellt sich die Sichtweise bei den Auftraggeberlnnen dar: $62 \%$ (fünf von acht) sehen Ihre Einrichtung als eine unter vielen, lediglich $25 \%$ (zwei von acht) als eine unter den wenigen wichtigen Nachfragern. Übereinstimmend ist das Bild dagegen hinsichtlich der Zusammensetzung der Auftragnehmerlnnen im Laufe der Jahre: Diese wechselten auch laut Einschätzung der AuftraggeberInnen zuletzt häufig (für $50 \%$ ) bzw. gelegentlich (für $37 \%$ ).

Während $25 \%$ der befragten Auftragnehmerlnnen den Wettbewerb auf nationaler Ebene als zuletzt gleichbleibend einschätzen, attestieren 
67 \% (acht von zwölf) eine verschärfte Wettbewerbssituation, wobei diese auf internationaler Ebene noch ausgeprägter wahrgenommen wird (82\%). Auch $88 \%$ (sieben von acht) der befragten Auftraggeberlnnen schätzen den Wettbewerb im Inland als sehr stark ein (vgl. Abb. 2).

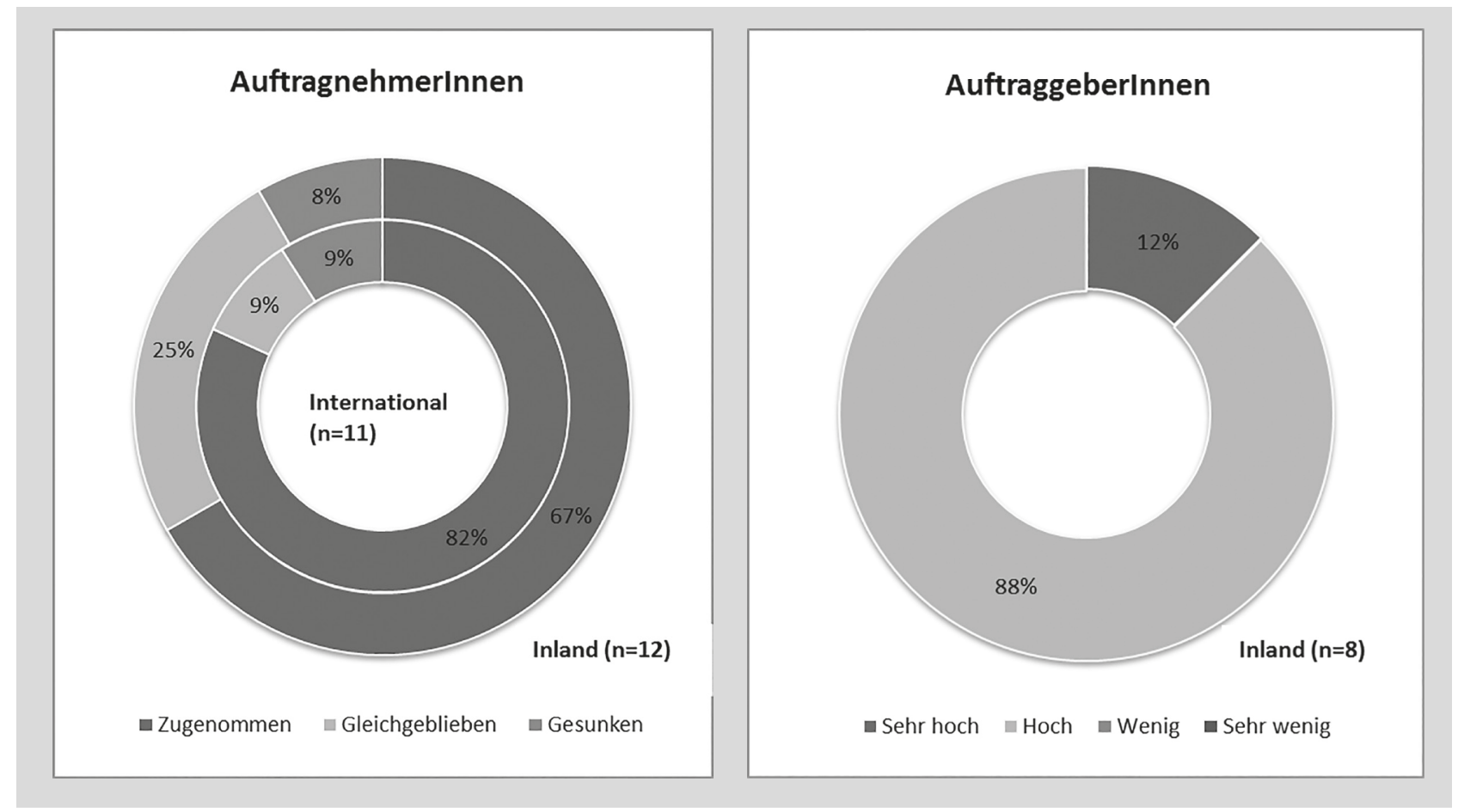

Abbildung 2: Einschätzung der Wettbewerbssituation, 2016-2018

Quelle: Online-Befragung Auftragnehmerlnnen und Auftraggeberlnnen 2019. Werte sind arithmetische Mittel.

Als Gründe für die verschärfte Wettbewerbssituation im Inland werden von den befragten Auftragnehmern am häufigsten der Markteintritt neuer MitbewerberInnen sowie ein stärkerer Preiswettbewerb genannt. Mit Blick auf die Entwicklungen der letzten fünf bis zehn Jahre wurden zudem steigende Anforderungen bei gleichbleibenden Budgets angeführt. Budgetäre Einschränkungen gehen laut Wahrnehmung der Auftragnehmerlnnen jedoch auch mit dem Verzicht auf weitreichendere, und somit kostenintensivere Fragestellungen in Leistungsbeschreibungen einher.

Das gezeichnete Bild deckt sich zum Großteil mit den Einschätzungen der Auftraggeberlnnen. Ein hoher Wettbewerb insbesondere bei kleineren Projekten steht einem zunehmenden Zeitdruck in der Verwaltung sowie budgetären Beschränkungen gegenüber. Einige Auftraggeberlnnen bestätigen, dass Anbieter aus anderen Ländern selten sind und nur bei größeren Projekten eine gewisse Rolle spielen. Auch die Professionalität der österreichischen Anbieter wird bestätigt. Ihre Kompetenz wird als „sehr hoch" (50 \%) bzw. „hoch“ (50 \%) eingeschätzt (jeweils vier von acht). Es findet sich auch die Wahrnehmung, dass bei Anbietern aus anderen Ländern keine höhere Qualität als bei österreichischen Anbietern festzustellen ist ${ }^{8}$.

\subsection{VERGABEPRAXIS}

Während Auftraggeberlnnen im Jahr 2016 im Schnitt 2,2 Teilnahmeanträge bzw. Angebote für einen zu vergebenden Evaluierungsauftrag erhielten, stieg die Zahl 2017 auf vier. 2018 ging der Wert wieder auf 3,5 zurück. Über diesen Zeitraum hinweg waren in den verschiedenen ausschreibenden Stellen jedoch teils große Schwankungen festzustellen. Dabei war in wenigen Fällen lediglich eine Einreichung pro Evaluierungsauftrag zu verzeichnen; in einem anderen Fall fanden acht Einreichungen den Weg zur ausschreibenden Stelle. Dieses Bild wird durch die Sekundärdatenanalyse im Großen und Ganzen bestätigt. Rund $90 \%$ der Teilnahmeanträge bzw. Angebote kamen von österreichischen Einrichtungen.

Im Durchschnitt über alle Befragten hinweg geben diese an, dass Evaluierungen mehrheitlich im Unterschwellenbereich vergeben wurden (vgl. Abb. 3). Dieses Ergebnis wird durch die Sekundärdatenanalyse gestützt. Hier konnten für denselben Zeitraum nur eine Handvoll EU-weit veröffentlichter Ausschreibungen (die also über dem Schwellenwert lagen) identifiziert werden. Da im Unterschwellenbereich angesiedelt, kam es in den allermeisten Fällen (zwei Drittel) zu Direktvergaben, deutlich 
weniger wurden in Form von Verhandlungsverfahren mit vorheriger Bekanntmachung (13\%) und offenen Verfahren (11\%) abgewickelt. Der Zuschlagswert, also jener Betrag, für den die Evaluierung schließlich beauftragt wurde, lag laut Auftraggeberlnnen in $80 \%$ der beauftragten Fälle in etwa gleichauf mit dem geschätzten Auftragswert.

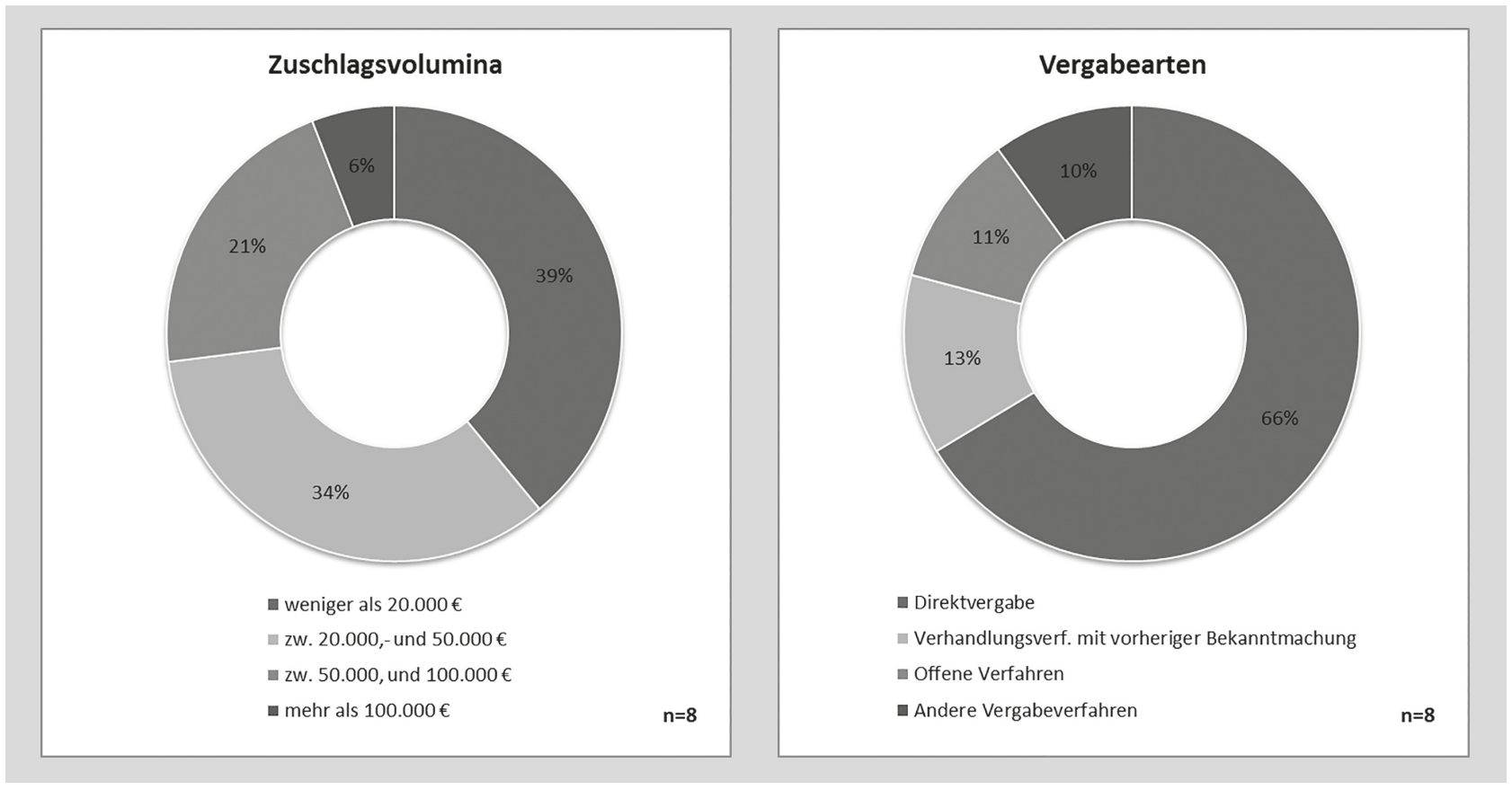

Abbildung 3: Zuschlagsvolumina und Vergabearten, 2016-2018

Quelle: Online-Befragung AuftraggeberInnen 2019. Werte sind arithmetische Mittel.

Gefragt nach den wichtigsten Herausforderungen bei Vergabeprozessen im Inland, nannten die Auftragnehmer am häufigsten sehr breite Anforderungen und detaillierte Fragestellungen sowie die Vorgabe enger budgetärer und zeitlicher Rahmen bei gleichzeitig hohen Anforderungen an methodische Tiefe und Innovation. Auf internationaler Ebene stellen vor allem Rahmenverträge, teils ,erzwungene' Konsortialbildung sowie lange Entscheidungshorizonte Herausforderungen dar. Aus Sicht der Auftraggeberlnnen liegen die Herausforderungen im Inland insbesondere darin, das für die Evaluierung verfügbare Budget mit der geplanten Aufgabenstellung in Einklang zu bringen, die Erstellung von Vergabeund Bewertungskriterien zu definieren und dabei das Vergaberecht einzuhalten.

\section{ZUSAMMENFASSUNG UND FAZIT}

Die vorliegende Studie liefert erste Einblicke in die Entwicklung des österreichischen Evaluierungsmarktes im FTI-Bereich in Bezug auf die MarktteilnehmerInnen, Wettbewerbssituation und Vergabepraxis. Dass im Zuge der Befragung ein Großteil der relevanten Marktteilnehmerlnnen erreicht wurde, spricht für eine hohe Validität der Ergebnisse, welche zudem im Rahmen einer Diskussionsveranstaltung der fteval Anfang Juli 2019 breite Zustimmung fanden. ${ }^{9}$

Die manchmal geäußerten Vermutungen, wonach der österreichische Evaluierungsmarkt von einer geringen Wettbewerbsintensität und einer vergleichsweise kleinen Zahl an Anbieterlnnen geprägt sei, konnten im Rahmen dieser Untersuchung nicht bestätigt werden. Vielmehr wird der Markt der FTI-Evaluierungen in Österreich sowohl von AuftraggeberInnen als auch Auftragnehmerlnnen als sehr wettbewerbsintensiv beschrieben. Dabei stellt der Markt die Auftragnehmer vor allem durch seine geringe Größe, eine wahrgenommene Konzentration aufseiten der Auftraggeber, den Markteintritt neuer Mitbewerberlnnen sowie wach- 
sende inhaltliche Anforderungen bei geringen Budgets vor große Herausforderungen.

Die Wettbewerbsintensität durch neue Anbieter ist hoch. Obwohl die Zahl der Mitglieder der Plattform fteval in den letzten Jahren gewachsen ist, entfällt ca. ein Viertel aller Aufträge auf Institutionen, die nicht Mitglieder der Plattform sind. In Richtung hoher Wettbewerbsintensität weisen auch die Antworten auf Fragen über die preisliche Wettbewerbsfähigkeit. Ein nicht unbeträchtlicher Teil der Marktteilnehmerlnnen bezeichnet die Projekte als oftmals nicht kostendeckend.

Die Untersuchung bestätigt teilweise die Vermutung über den geringen Internationalisierungsgrad des österreichischen Evaluierungsmarktes. Es ist zu vermuten, dass der österreichische Evaluierungsmarkt mit seiner insgesamt relativ geringen Größe, den geringen Projektgrößen, und der wachsenden Zahl an Mitbewerbern für ausländische Anbieter nur begrenzt attraktiv ist. Hinzu kommt eventuell das Spezifikum von Deutsch als Berichtssprache. Die Nachfrage ist von Direktvergaben gekennzeichnet; diese sind oft weniger transparent als andere Vergabeformen. Anbieter aus anderen Ländern sind kaum im Lead von Konsortien zu finden, und auch unter den Konsortialmitgliedern schwach vertreten.

Auf der Seite der von den Auftraggeberlnnen wahrgenommenen Evaluierungskompetenz der österreichischen Anbieter dürfte es kein Problem geben: Diese wird durchwegs - auch im Vergleich mit ausländischen Anbietern - als hoch bzw. sehr hoch eingeschätzt. Zudem sind österreichische Anbieter von Evaluierungen vielfach auch auf internationaler Ebene aktiv.

Insgesamt weisen die vorliegenden Befunde auf einen wettbewerbsintensiven FTI-Evaluierungsmarkt in Österreich hin, der aber zum allergrößten Teil von einer Vielzahl österreichischer Anbieterlnnen bedient wird, denen eine hohe Qualität bescheinigt wird. Gleichzeitig weist er einige Charakteristika auf, die sich negativ auf eine qualitative Entwicklung auswirken können: geringe Projektgrößen, hoher Preisdruck und ein hoher Anteil an Direktvergaben könnten solche potentiell retardierende Faktoren sein. Versuche, den Markt für FTI-Evaluierungen qualitativ weiter zu entwickeln, sollten hier ansetzen.

Abschließend ist nochmals zu betonen, dass die gegenständliche Studie nicht alle Dimensionen des FTI-Evaluierungsmarktes, sondern nur den klar abgegrenzten Bereich der Vergabe öffentlicher Aufträge für wissenschaftliche Dienst- und Forschungsleistungen abdecken konnte. Darüber hinausgehende Fragen, etwa zur Bedeutung von Studien, Analysen und wissenschaftlichen Begleitaktivitäten, die nicht Evaluierungen entsprechend der hier zugrunde gelegten Definition umfassen, bedürfen näherer Untersuchungen. Gleichwohl hoffen wir, mit dieser Studie eine (erste) empirische Basis für eine fundierte Diskussion gelegt zu haben. Eine anzustrebende wichtige Erweiterung der Analyse wäre ein Vergleich des österreichischen Marktes mit anderen Ländern.

\section{REFERENZEN}

Aiginger, K., Falk, R. und Reinstaller A. (2009): Evaluation of government funding in RTDI from a systems perspective in Austria. Synthesis Report, Vienna.

Biegelbauer, P. (2013). Wie lernt die Politik? Lernen aus Erfahrung in Politik und Verwaltung. Springer-Verlag.
BMBWF, BMVIT und BMDW (2018): Österreichischer Forschungs- und Technologiebericht 2018. Bericht der Bundesregierung an den Nationalrat gem. § 8 (2) FOG über die Lage und Bedürfnisse von Forschung, Technologie und Innovation in Österreich, Wien.

BMWFW und BMVIT (2017): Österreichischer Forschungs- und Technologiebericht 2017. Lagebericht gem. § 8 (1) über die aus Bundesmitteln geförderte Forschung, Technologie und Innovation in Österreich, Wien.

Böttcher, W., Kerlen, C., Maats, P., Schwab, O., \& Sheikh, S. (2014). Evaluation in Deutschland und Österreich. Münster: Waxmann.

DeGEval Standards (2016): DeGEval -Gesellschaft für Evaluation. Standards für Evaluation. Erste Revision auf Basis der Fassung 2002. https:// www.degeval.org/fileadmin/Publikationen/DeGEval_Standards_fuer_ Evaluation_-_Erste_Revision_2016_.pdf

Dinges, M. und Schmidmayer, J. (2010): Country report: Austria., in: Edler, J., Cunningham, P., Gök, A., Rigby, J., Amanatidou, E., Garefi, I., Bührer, S., Dinges, M., Berger, M., Schmid-mayer, J. und Guy, K. (2010): Understanding evaluation of innovation policy in Europe, in: INNO-Appraisal final report, Manchester, 243-264

fteval Standards (2019): Standards der Evaluierung in der Forschungs-, Technologie- und Innovationspolitik. https://repository.fteval.at/386/7/ standards_DT_WEB_08042019.pdf

Handler, H. (2005): Öffentliches Auftragswesen und Wettbewerb im gesamtwirtschaftlichen Zusammenhang; in: Michael Sachs (Hrsg.), Schwerpunkte zum BVergG 2006: Ökonomische und rechtliche Aspekte, Wien (Manz) 2005: 27-58.

Landsteiner, G. (2015): Nützlichkeit und Nutzen der Programmevaluationen im Bereich der österreichischen FTI-Politik. Metaevaluation der Programmevaluationen 2003-2014

Reiner, D. und Smoliner S. (2012): Outputorientierte Evaluierung öffentlich geförderter FTI-Programme. Studie im Auftrag des Bundesministeriums für Verkehr, Innovation und Technologie, Wien

Streicher, J. (2017): Evaluations, Actors and Institutions. The Case of Research, Technology and Innovation Policy in Austria

Tsipouri, L und Sidiropolous, N. (2014): RTDI evaluation culture in the EVAL-INNO coun-tries. In: Zupan I, Gajdusek MF and Marinkovic I (Hsg.) Fostering Evaluation Competencies in Research, Technology and Innovation - the EVAL-INNO experience, S. 11-20. LIT Verlag.

Warta, K. und Philipp. S. (2016): Was bringt die Plattform fteval und zu was hat sie es ge-bracht? Rückblicke und Ergebnisse einer Mitgliederbefragung, The fteval Journal for Rese-arch and Technology Policy Evaluation, Ausgabe 41, 5-14 


\section{ABBILDUNGSVERZEICHNIS}

Abbildung 1: $\quad$ Anzahl an identifizierten Evaluierungen im Zeitraum 2009-2018 76

Abbildung 2: $\quad$ Einschätzung der Wettbewerbssituation, 2016-2018 78

Abbildung 3: $\quad$ Zuschlagsvolumina und Vergabearten, 2016-2018 79

\section{TABELLENVERZEICHNIS}

Tabelle 2: $\quad$ Evaluierungen auf nationaler Ebene, 2002-2007 sowie 2016-2018

\section{AUTOREN}

JÜRGEN STREICHER

Joanneum Research

Sensengasse 1, 1090 Wien

E: juergen.streicher@joanneum.at

WOLFGANG POLT

Joanneum Research

Sensengasse 1, 1090 Wien

E: wolfgang.polt@joanneum.at

MAXIMILIAN UNGER

Joanneum Research

Sensengasse 1, 1090 Vienna

E. maximilian.unger@joanneum.at

\section{KEYWORDS:}

Evaluierungsmarkt; FTI-Evaluierung; Österreich 